Rev. Int. Contam. Ambie. 36 (1) 55-62, 2020

DOI: $10.20937 /$ RICA.2020.36.53423

\title{
DISEÑO Y ENSAYO DE FIBRAS PLÁSTICAS RECICLADAS PARA REFUERZO DE MORTERO
}

Design and test of recycled plastic fibers for mortar reinforcement

\author{
Juan Pablo OJEDA*, Irma Teresa MERCANTE y Nicolás Horacio FAJARDO
}

Centro de Estudios de Ingeniería de Residuos Sólidos, Instituto de Medio Ambiente, Facultad de Ingeniería, Universidad Nacional de Cuyo, Centro Universitario (M5502KFA), Casilla de correo 405, Mendoza, Argentina *Autor para correspondencia: juanpabloojedadangelo@hotmail.com

(Recibido: octubre 2018, aceptado: junio 2019)

Palabras clave: reciclaje, residuos, cemento, mezclas

\section{RESUMEN}

El incremento en la generación de residuos sólidos supone un desafío para el desarrollo de nuevas formas de reciclaje y reaprovechamiento de los materiales. Los plásticos constituyen el $10 \%$ de los residuos sólidos a nivel mundial y pueden emplearse para la fabricación de fibras para hormigones y morteros. El objetivo de este trabajo fue diseñar y ensayar fibras plásticas recicladas incorporadas en mortero de cemento y comparar su comportamiento con el de fibras comerciales. Para ello se elaboraron tres dosificaciones: una mezcla patrón, otra mezcla con las fibras diseñadas y otra con fibras comerciales. Se construyeron probetas y se realizaron ensayos de resistencia a flexión y a compresión. La mezcla con las fibras desarrolladas logró más de una vez y media la resistencia a flexión de la mezcla patrón a 28 días, contra sólo un $25 \%$ de aumento en el caso de la mezcla con fibras comerciales. La resistencia a la compresión también se mejoró. Se concluyó que el desempeño de las fibras diseñadas fue mejor que el de las fibras comerciales y que el uso de fibras mejoró las propiedades estudiadas respecto de morteros sin fibras.

Key words: recycling, waste, cement, mixes

\begin{abstract}
The generation increase of solid waste represents a challenge for the development of new forms of recycling and reuse of materials. Plastics make up $10 \%$ of solid waste worldwide and can be used to manufacture fibers for concrete and mortars. The aim of this paper was to design, and test recycled plastic fibers incorporated in cement mortars and compare their behavior with that of commercial fibers. For this, three dosages were elaborated: one standard mix, another mix with the designed fibers and a third one with commercial fibers. Test specimens were constructed and mechanical tests of compressive and flexural strength were carried out. The mixture with the developed fibers achieved more than one and a half times the flexural strength of the standard mixture at 28 days, while only a $25 \%$ increase in the case of commercial fibers mixtures was achieved. The compressive resistance also improved. It was concluded that the performance of the designed fibers was better than that of the commercial fibers, and that the use of fibers improved the properties studied with respect to mortars without fibers.
\end{abstract}




\section{INTRODUCCIÓN}

El hormigón es uno de los materiales de construcción de uso más extendido en el mundo. Está compuesto por cemento portland, agregados y agua, aunque también puede contener aditivos, adiciones minerales y fibras de refuerzo. La industria del hormigón es, también, una de las mayores consumidoras de energía y de materia prima virgen.

Diversos investigadores han propuesto utilizar materiales reciclados para reemplazar algunos de sus componentes. Como agregados pueden usarse escorias o residuos de demolición; las escorias de alto horno, las cenizas volantes y el vidrio molido pueden sustituir parcialmente el cemento, y las fibras de acero, vidrio o plástico pueden incorporarse para reforzar el hormigón (Ojeda et al. 2017).

Por otro lado, cada vez es mayor la preocupación sobre el tema de los residuos sólidos. Para el año 2012 se generaron 1300 millones t/año de residuos y se espera que para 2025 aumenten a aproximadamente 2200 millones t/año debido al aumento de las tasas de generación de desechos per cápita, de 1.2 a $1.42 \mathrm{~kg}$ por persona por día (Hoornweg y Bhada-Tata 2012).

La fracción de residuos plásticos constituye un $10 \%$ de todos los residuos sólidos urbanos (RSU) a nivel global (Hoornweg y Bhada-Tata 2012). De allí la oportunidad de emplear estos materiales para producir fibras para el hormigón.

Los residuos plásticos pueden reciclarse mediante diversos procesos. Pueden aplicarse procesos de lavado, corte, extrusión, conformado, entre otros, para diversas necesidades: control de fisuración, mejor resistencia a flexión, hormigones proyectados, resistencia a la abrasión, etc.

Foti et al. (2011) utilizaron fibras rectas y anillos cortados de botellas de polietileno tereftalato (PET), obteniendo un hormigón más dúctil. Foti et al. (2013) estudiaron el comportamiento del PET como reemplazo del acero de refuerzo en el hormigón armado, consiguiendo buenos resultados y concluyendo que es posible realizar esta sustitución. Fraternali et al. (2011) investigaron la conductividad térmica, la resistencia a la compresión, la resistencia a la flexión y la ductilidad de hormigones con $1 \%$ de fibras PET recicladas y extruidas con distintas geometrías. Obtuvieron marcadas mejoras en las resistencias térmica y mecánica, así como mejor ductilidad.

En cuanto a la resistencia química de las fibras, Ochi et al. (2007) concluyeron que las fibras de PET no sufren ataque de los álcalis en hormigones convencionales. Luego, Fraternali et al. (2013) confirmaron estas conclusiones. Won et al. (2010) estudiaron la durabilidad de las fibras PET en ambientes alcalinos, ciclos de congelamiento y deshielo y otros ambientes químicos agresivos, incluyendo $\mathrm{NaCl}, \mathrm{CaCl}_{2}, \mathrm{Na}_{2} \mathrm{SO}_{4}$ y $\mathrm{H}_{2} \mathrm{SO}_{4}$. Concluyeron que no hubo afectación de las fibras.

Ghernouti et al. (2015) utilizaron fibras obtenidas a partir del reciclaje de bolsas plásticas en hormigones autocompactantes, concluyendo que estas no desmejoraban las propiedades en estado fresco de estas mezclas, ni las propiedades mecánicas en estado endurecido, pero mejoraban la resistencia a tracción por compresión diametral.

Kim et al. (2008) compararon el efecto en la fisuración por contracción del hormigón debido a diferentes morfologías de fibras: rectas, onduladas y con textura, obteniendo el mejor desempeño las de textura.

Kim et al. (2010) estudiaron el efecto de fibras PET y concluyeron que la resistencia a la compresión y el módulo de elasticidad caen a medida que aumenta el porcentaje de fibras, mientras que la fisuración por contracción y la ductilidad se ven mejoradas. Pereira de Oliveira et al. (2011) concluyeron que en morteros reforzados con hasta 1.5 $\%$ de fibras PET, la resistencia a la flexión se ve incrementada. Spadea et al. (2015) estudiaron fibras de nailon recicladas a partir de redes de pesca como refuerzo de morteros de cemento en contenidos de $1 \%$ y $1.5 \%$. Concluyeron que se incrementó la resistencia a la tracción en hasta $35 \%$ y la tenacidad hasta 13 veces respecto del mortero sin fibras. Yin et al. (2015a) estudiaron fibras plásticas de polipropileno (PP) virgen y reciclado con textura, con un contenido de $4 \mathrm{~kg} / \mathrm{m}^{3}$. Concluyeron que las fibras de material virgen resistieron más a la tracción y presentaron un módulo de Young inferior que el de las fibras recicladas. Aun así, las fibras recicladas tuvieron buen desempeño posterior a la fisuración y no afectaron la resistencia a la compresión.

La investigación sobre el desempeño de fibras plásticas en hormigones, y especialmente en morteros, aún debe ser profundizada, pues las fibras no son ampliamente utilizadas por las industrias de la construcción. A su vez, deben realizarse investigaciones de alcance local para estudiar las características particulares de los materiales, principalmente de los agregados y el comportamiento de las mezclas.

El objetivo de este trabajo fue diseñar fibras a partir de residuos plásticos para incluirlas en mortero, ensayar su comportamiento mecánico a compresión y flexión y comparar los resultados con los producidos por el uso de fibras comerciales de nailon. 
CUADRO I. MATERIALES Y CARACTERÍSTICAS

\begin{tabular}{|c|c|c|c|c|}
\hline Componente & Material & Características & & \\
\hline \multirow[t]{2}{*}{ Agregados } & Arena fina & TMN $0.59 \mathrm{~mm}$ & MF 1.5 & \multirow{2}{*}{$\begin{array}{l}\text { Arenas naturales silíceas } \\
\text { de depósito aluvial }\end{array}$} \\
\hline & Arena gruesa & TMN $2.4 \mathrm{~mm}$ & MF 2.7 & \\
\hline \multirow[t]{2}{*}{ Fibras } & \multicolumn{4}{|c|}{ Diseñadas y presentadas en los resultados } \\
\hline & $\begin{array}{l}\text { Comerciales de } \\
\text { Nailon }\end{array}$ & Densidad relativa 1.15 & \multicolumn{2}{|c|}{ Largo: 200 mm. Diámetro: $80 \mu \mathrm{m}$. } \\
\hline \multicolumn{2}{|l|}{ Agua } & \multicolumn{3}{|l|}{ Agua potable } \\
\hline Ligante & $\begin{array}{l}\text { Cemento Portland } \\
\text { compuesto }\end{array}$ & Resistencia 40 & \multicolumn{2}{|c|}{$\begin{array}{l}\text { Compuesto con mezcla de puzolana } \\
\text { natural y filler calcáreo. }\end{array}$} \\
\hline
\end{tabular}

TMN = tamaño máximo nominal del agregado, $\mathrm{MF}=$ módulo de finura de los agregados

\section{MATERIALES Y MÉTODOS}

\section{Caracterización de los materiales de la mezcla}

Los materiales utilizados y sus características se presentan en el cuadro I. Los componentes de la mezcla se seleccionaron de acuerdo a mezclas de cemento utilizadas por una empresa constructora local.

\section{Dosificación}

En la figura 1 se presentan las curvas granulométricas de las arenas, la mezcla de arenas y las curvas límites (A, B y C) establecidas en el reglamento del Centro de Investigación de los Reglamentos Nacionales de Seguridad para las Obras Civiles (CIRSOC) 201 (INTI 2015). Se diseñaron mezclas utilizando dos tipos de arena (denominadas arena fina y arena gruesa) en una proporción fija de aproximadamente 3 a 7 de arena fina y arena gruesa, respectivamente, a los fines de mantenerse dentro de las curvas.
De acuerdo con CIRSOC 201 (INTI 2015), el módulo de finura (MF) debe estar comprendido entre 2.3 y 3.1. Según la dosificación utilizada, la mezcla de arenas posee 2.34 de MF.

La curva A presenta el límite inferior de la granulometría y la B el límite superior. Para mezclas con resistencias especificadas menores a $20 \mathrm{MPa}$, se amplía la zona granulométrica hasta la curva $\mathrm{C}$.

El contenido de cemento se fijó en $366 \mathrm{~kg} / \mathrm{m}^{3}$ y la relación agua cemento fue de 0.55 para todos los casos. El agua se obtuvo a partir de esta relación, dosificando $201.3 \mathrm{~kg} / \mathrm{m}^{3}$. Finalmente, se calculó la cantidad de agregados naturales necesaria para completar la dosificación.

En el cuadro II pueden observarse las dosificaciones de las mezclas a (patrón), b (fibras PET) y c (fibras nailon).

La dosificación recomendada por el fabricante de fibras comerciales es de $0.6 \mathrm{~kg} / \mathrm{m}^{3}$ pero, que estas

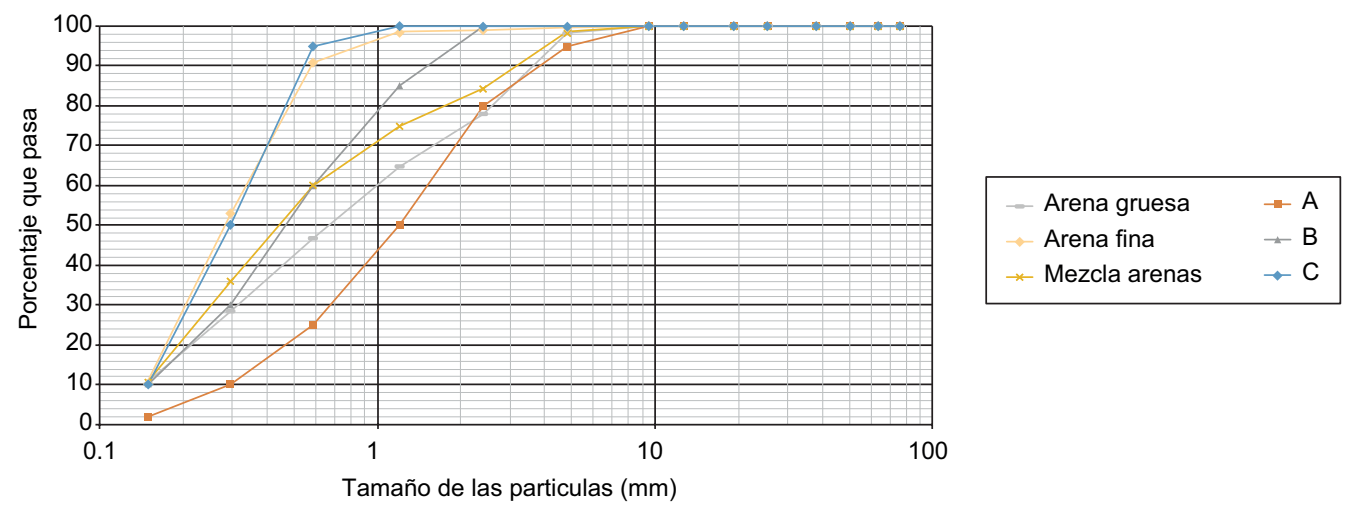

Fig. 1. Curvas granulométricas: A, B y C del Centro de Investigación de los Reglamentos Nacionales de Seguridad para las Obras Civiles 201 (INTI 2015), arena fina, arena gruesa y mezcla de arena 
CUADRO II. DOSIFICACIONES

\begin{tabular}{lccc}
\hline Mezcla $\left(\mathrm{kg} / \mathrm{m}^{3}\right)$ & $\mathrm{a}$ & $\mathrm{b}$ & $\mathrm{c}$ \\
\hline Arena fina & 470 & 468.7 & 464.5 \\
Arena gruesa & 1083 & 1080.8 & 1071 \\
Cemento & 366 & 366 & 366 \\
Agua & 201.3 & 201.3 & 201.3 \\
Fibras & - & 1.5 & 9.4 \\
\hline
\end{tabular}

fibras se utilizan para controlar la formación de fisuras, se aumentó la dosificación para evaluar su desempeño a la flexión.

\section{RESULTADOS Y DISCUSIÓN}

\section{Diseño de fibras}

Se realizó una búsqueda bibliográfica de antecedentes sobre el uso de fibras plásticas de origen residual en hormigones. Luego, se propuso el diseño de las fibras (ancho, largo, espesor, morfología) y el tipo de material a emplear, considerando los valores más frecuentes de 17 publicaciones recientes (menos de 10 años de antigüedad), obtenidas de revistas científicas con arbitraje (Ochi et al. 2007, Kim et al. 2008, Kim et al. 2010, Won et al. 2010, Foti 2011, Fraternali et al. 2011, Pereira-deOliveira y Castro-Gomes 2011, Pereira-de-Oliveira et al. 2012, Foti 2013, Fraternali et al. 2013, Fraternali et al. 2014, Koo et al. 2014, Ghernouti et al. 2015, Spadea et al. 2015, Yin et al. 2015a, Yin et al. 2015b y Borg et al. 2016).

Se construyeron los gráficos que se observan en las figuras 2,3 y 4 . La figura 2 muestra la distribución de frecuencias de longitudes para cada rango de tamaños analizados. La figura 3 señala la distribución de frecuencias de ancho/diámetro para distintos rangos de tamaños. La figura 4 presenta la distribución de frecuencias de cada tipo de plástico encontrado en la bibliografía consultada.

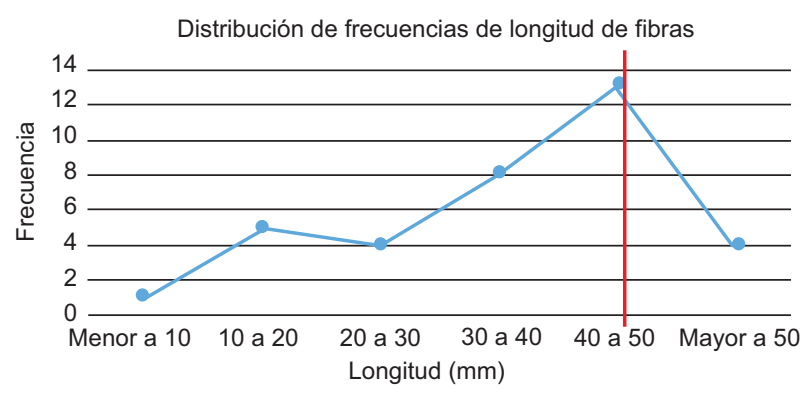

Fig. 2. Frecuencias de longitudes de las fibras. La línea roja indica el intervalo más frecuente

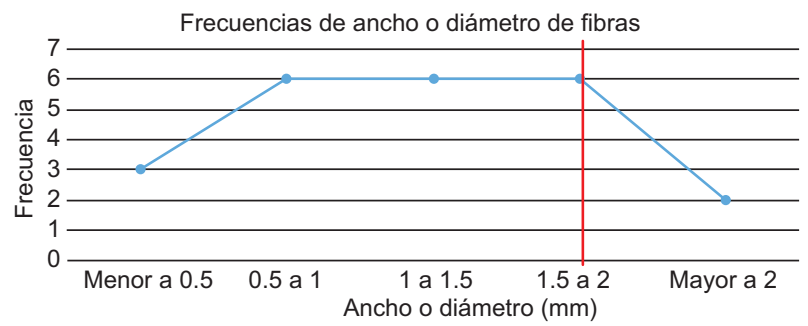

Fig. 3. Frecuencias de ancho o diámetro de las fibras. La línea roja indica el intervalo más frecuente

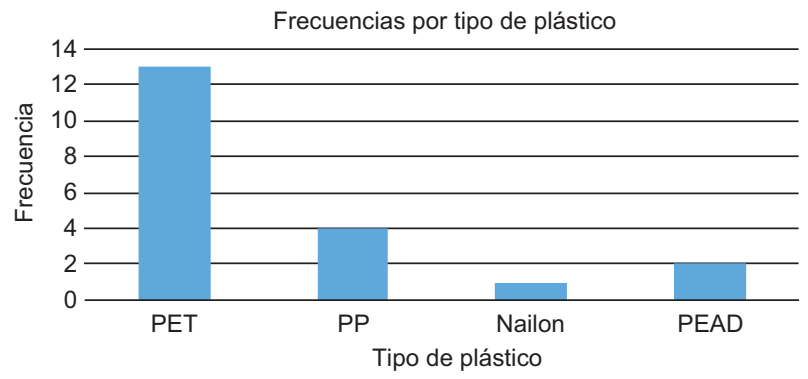

Fig. 4. Frecuencias por tipo de plástico: polietileno tereftalato (PET), polipropileno (PP), nailon y polietileno de alta densidad (PEAD)

En función de los resultados obtenidos se escogió un largo de $45 \mathrm{~mm}$ y un ancho de $2 \mathrm{~mm}$, no sólo por ser los valores más frecuentes en las investigaciones, sino también pensando en la practicidad de la fabricación de las fibras. En este caso fueron cortadas manualmente, pero se podría industrializar el proceso.

Como se muestra en la figura 4, el PET es el más frecuentemente utilizado y por lo tanto se eligió para el diseño de las fibras. Se emplearon botellas de bebidas post-consumo para obtener las fibras. El espesor fue el de la pared de las botellas de bebidas $(0.35 \mathrm{~mm})$.

El comportamiento de las fibras puede optimizarse mediante tratamientos superficiales o conformado para mejorar la adherencia con el mortero (Yin et al. 2015a). Sin embargo, en estos ensayos se adoptó la forma recta y sin textura superficial.

En la figura 5 pueden observarse las fibras utilizadas, junto a la escala gráfica con graduación de $1 \mathrm{~cm}$.

\section{Ensayos realizados}

Se confeccionaron probetas para ensayo de flexión y compresión a siete y 28 días. Los moldes empleados corresponden a los establecidos por el Instituto Argentino de Normalización y Certificación (IRAM) en la norma 1622 (IRAM 2015). El curado se hizo sumergiendo en agua saturada con cal según norma 1534 (IRAM 2018). 


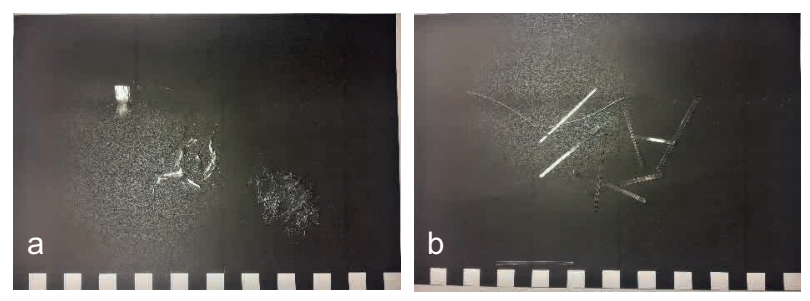

Fig. 5 Fibras junto a escala de $1 \mathrm{~cm}$ de: a) nailon, b) polietileno terftalato (PET)

Los ensayos mecánicos se realizaron en los laboratorios del Centro de Estudios de Ingeniería de Residuos Sólidos (CEIRS) y el Instituto Técnico de Investigaciones y Ensayo de Materiales (ITIEM) de la Facultad de Ingeniería, Universidad Nacional de Cuyo. Para efectuar los ensayos mecánicos se utilizó una máquina universal de ensayos Amsler con capacidad máxima de $30000 \mathrm{~kg}$ y resolución de $10 \mathrm{~kg}$, calibrada conforme a la norma ISO 376.

\section{Flexión}

Para cada edad de ensayo se elaboraron tres probetas prismáticas de $4 \mathrm{~cm} \times 4 \mathrm{~cm} \times 16 \mathrm{~cm}$ y se las ensayó a flexión. Durante el ensayo se sometió a la probeta a una carga gradual y localizada en su mitad, hasta su rotura. Se utilizó una máquina universal que se acondicionó para este ensayo, como se observa en la figura 6.

Se determinó la resistencia a la flexión mediante la ecuación 1.

$R_{f}=\frac{1.5 \times F_{t} \times l}{b^{3}}$

Donde:

$R_{f}$ : resistencia a la flexión en $\mathrm{MPa}$.

$F_{t}$ : carga de rotura en $\mathrm{N}$.

$l$ : luz de ensayo en $\mathrm{mm}$.

$b$ : ancho de la probeta en $\mathrm{mm}$.

Se obtuvieron tres valores de resistencia a $7\left(\mathrm{Rf}_{7}\right.$ días $)$ y 28 días $\left(\mathrm{Rf}_{28 \text { días }}\right)$ y se adoptó como resistencia a la flexión de cada mezcla el promedio de dichos valores (Cuadro III).

El mejor desempeño obtenido fue el de la mezcla $b$, con fibras PET. Si bien a 7 días la mezcla patrón (a) tuvo mayor resistencia que la mezcla con fibras comerciales (c), a 28 días la mezcla c superó la resistencia de la mezcla patrón.

A 28 días, las mezclas b y c tuvieron $150 \%$ y $125 \%$ de la resistencia de la mezcla patrón a flexión. A 7 días, el porcentaje de ganancia de resistencia a la flexión respecto del de 28 días fue $91 \%$ para la mezcla

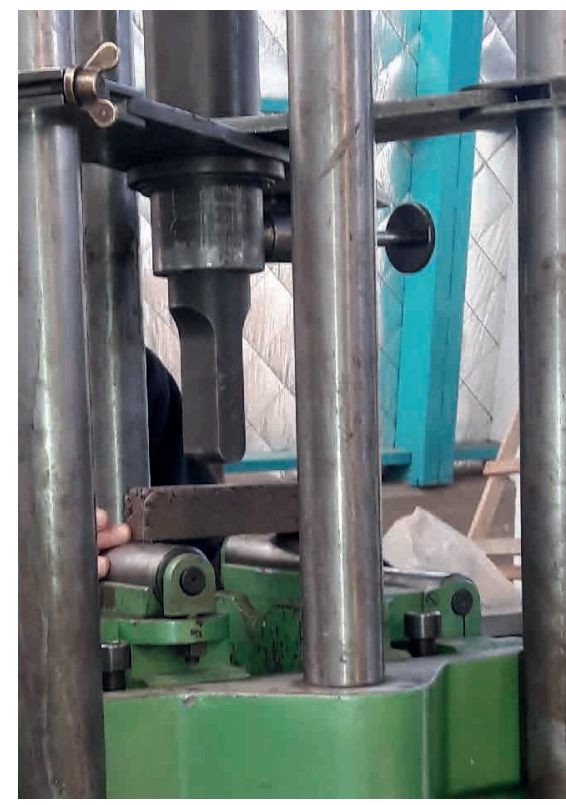

Fig. 6. Ensayo a flexión

CUADRO III. RESISTENCIAS A FLEXIÓN (Rf). PROBETAS PRISMÁTICAS

\begin{tabular}{llllll}
\hline $\begin{array}{l}\text { Denominación } \\
\text { de la probeta }\end{array}$ & \multicolumn{2}{c}{$\begin{array}{l}\mathrm{Rf}_{7 \text { días }} \\
(\mathrm{MPa})\end{array}$} & \multicolumn{2}{c}{$\begin{array}{c}\mathrm{Rf}_{28 \text { días }} \\
(\mathrm{MPa})\end{array}$} \\
\hline & 1 & 1.97 & & $---{ }^{2}$ & \\
a & 2 & 1.97 & 1.90 & 2.19 & 2.08 \\
& 3 & 1.75 & & 1.97 & \\
\hline & 1 & 1.97 & & 2.91 & \\
b & 2 & 2.19 & 2.08 & 3.57 & 3.50 \\
& 3 & ---- & & 4.01 & \\
\hline & 1 & 1.08 & & 2.03 & \\
c & 2 & 1.08 & 1.01 & ---- & 2.58 \\
& 3 & 0.86 & & 3.13 & \\
\hline
\end{tabular}

$\mathrm{MPa}=$ megapascal. Nota: las celdas con ausencia de valores corresponden a probetas que no pudieron ensayarse a flexión porque se partieron antes del ensayo. Aun así, las mitades obtenidas se ensayaron a compresión.

a, $60 \%$ para la mezcla b y $40 \%$ para la mezcla c. Esto indica que a mayor contenido de plástico, más lenta es la ganancia de resistencia a flexión.

El tipo de falla obtenido para las mezclas con fibras (b y c) fue de arrancamiento. Esto podría deberse a la ausencia de una textura superficial que mejore la adherencia entre el plástico y la pasta de cemento. También podrían ensayarse distintos contenidos de cada tipo de fibras, para obtener conclusiones respecto del efecto de un mayor porcentaje de fibras en las propiedades mecánicas del mortero. 


\section{Compresión}

Las seis mitades obtenidas por rotura de los especímenes a flexión fueron ensayadas a compresión para cada edad. Debido a que las muestras ya se encontraban alteradas por el ensayo previo, también se ensayaron a compresión tres probetas cúbicas de $7 \mathrm{~cm} \times 7 \mathrm{~cm} \times 7 \mathrm{~cm}$ a los 28 días (Fig. 7).

En el proceso de ensayo, mediante la máquina universal, se aplicaron cargas de manera gradual y uniforme en el área de la probeta. En el caso de las mitades de probetas prismáticas se empleó un dispositivo para aplicar la carga exclusivamente en un área de $1600 \mathrm{~mm}^{2}$, mientras que en la cúbica se tomó toda el área $\left(4900 \mathrm{~mm}^{2}\right)$.

Se determinó la resistencia a la compresión por medio de la ecuación 2.

$R_{c}=\frac{F_{c}}{A}$

Donde:

$\mathrm{R}_{\mathrm{c}}$ : resistencia a la compresión en $\mathrm{MPa}$.

$\mathrm{F}_{\mathrm{c}}$ : carga de rotura en $\mathrm{N}$.

A: área determinada de cada probeta en $\mathrm{mm}^{2}$.

La resistencia a la compresión de las probetas prismáticas se obtuvo promediando el resultado de las dos mitades de las probetas ensayadas a flexión a 7 ( $\left.\mathrm{Rpc}_{7 \text { días}}\right)$ y 28 días ( $\left.\mathrm{Rpc}_{28 \text { días}}\right)$; y luego promediando los tres valores así obtenidos (Cuadro IV).

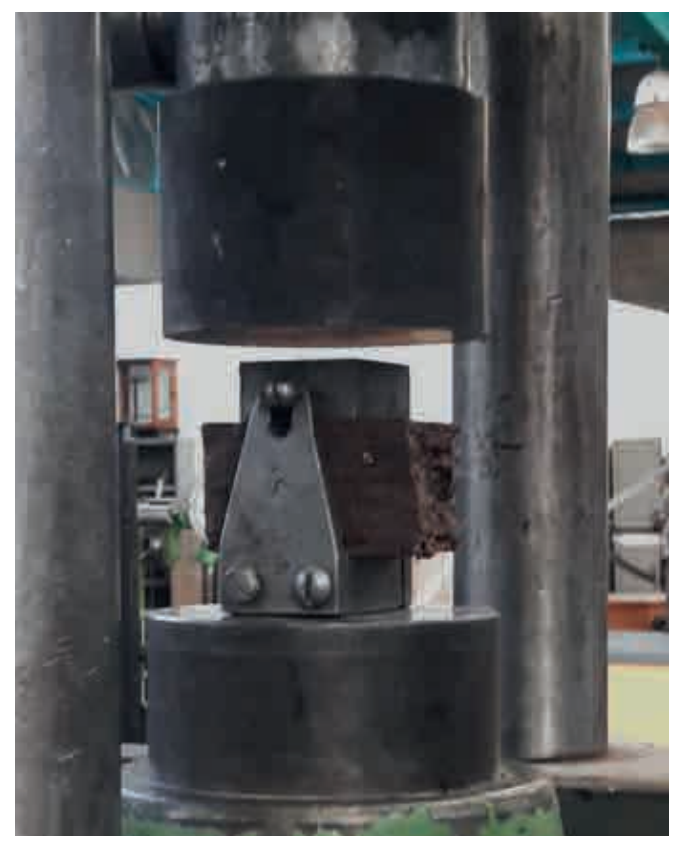

Fig. 7. Ensayo a compresión sobre probeta prismática

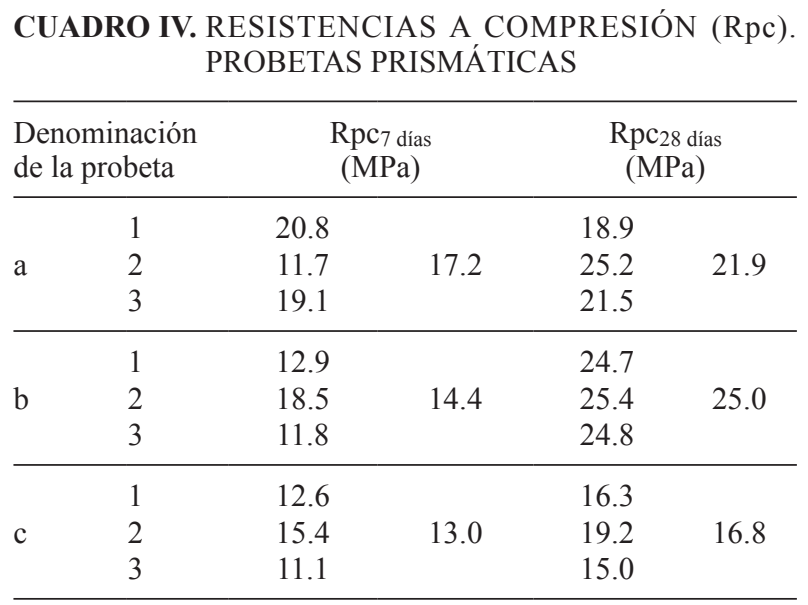

$\mathrm{MPa}=$ megapascal

La resistencia a la compresión de las tres probetas cúbicas a 28 días ( $\operatorname{Rcc}_{28}$ días) se obtuvo como el promedio de los tres resultados de ensayo. En el cuadro V se presentan estos resultados.

Se observaron mejoras en la resistencia a la compresión a 28 días con el uso de las fibras PET, algo que no coincide con lo observado por Kim et al. (2010). Por otro lado, sí se encontró una disminución de la resistencia a compresión debido al uso de fibras de nailon, tanto a siete como a 28 días.

La resistencia a los siete días de la mezcla b obtuvo un $58 \%$ de la resistencia de diseño (28 días), en cambio la mezcla c alcanzó un $80 \%$ de la resistencia final a la misma edad.

Todas las resistencias a la compresión obtenidas a partir de las probetas prismáticas partidas fueron mayores que las de las probetas cúbicas. Esto se debe a que las probetas prismáticas son de menor

CUADRO V. RESISTENCIAS A COMPRESIÓN (Rcc). PROBETAS CÚBICAS

\begin{tabular}{llll}
\hline Denominación & \multicolumn{2}{c}{$\operatorname{Rcc}_{28 \text { días }}(\mathrm{MPa})$} \\
\hline & 1 & 19.2 & \\
$\mathrm{a}$ & 2 & 24.8 & 21.3 \\
& 3 & 20.0 & \\
\hline & 1 & 23.4 & 22.9 \\
$\mathrm{~b}$ & 2 & 20.2 & \\
& 3 & 25.2 & \\
\hline & 1 & 18.4 & \\
$\mathrm{c}$ & 2 & 15.1 & \\
& 3 & 15.4 &
\end{tabular}

$\mathrm{MPa}=$ megapascal 
tamaño relativo. Además, se observó la ocurrencia de una rotura por corte en los bordes del aplicador de cargas, lo que podría explicar también el incremento en la resistencia.

\section{CONCLUSIONES}

Se diseñaron fibras utilizando residuos PET de botellas de bebida. Las dimensiones de las fibras diseñadas fueron de $45 \mathrm{~mm}$ de largo, $2 \mathrm{~mm}$ de ancho y $0.35 \mathrm{~mm}$ de espesor. No se imprimió ninguna textura superficial a las fibras, por lo que el tipo de falla observado en la flexión fue de arrancamiento. Con el empleo de alguna textura superficial podría mejorarse el desempeño de las fibras.

El mejor desempeño obtenido fue el de la mezcla b, con fibras PET. Si bien a 7 días la mezcla patrón (a) tuvo mayor resistencia que la mezcla con fibras comerciales (c), a 28 días la mezcla c superó la resistencia de la mezcla patrón. La mejora de la resistencia a flexión a 28 días fue de $150 \%$ y $125 \%$ para las mezclas b y c, respectivamente.

La mezcla c fue la que más lentamente ganó resistencia, seguida de la mezcla b. Se concluye que el aumento del contenido de plástico produce una disminución en la ganancia de resistencia a la flexión a edades tempranas, respecto de la resistencia final.

A siete días la mezcla a fue la que mejor resistencia tuvo. Sin embargo, a 28 días la mezcla b superó la resistencia a la compresión de las mezclas a y c.

Los resultados obtenidos permitieron concluir que el uso de fibras PET mejoró el comportamiento mecánico a la compresión, contradiciendo lo presentado por Kim et al. (2010). El empleo de las fibras comerciales de nailon sí produjo caída de la resistencia a la compresión.

\section{AGRADECIMIENTOS}

A la Secretaría de Investigación, Internacionales y Posgrado de la Universidad Nacional de Cuyo por el financiamiento otorgado.

\section{REFERENCIAS}

Borg R. P., Baldacchino O. y Ferrara L. (2016). Early age performance and mechanical characteristics of recycled PET fibre reinforced concrete. Constr. Build. Mater. 108, 29-47. DOI: 10.1016/j.conbuildmat.2016.01.029
Foti D. (2011). Preliminary analysis of concrete reinforced with waste bottles PET fibers. Constr. Build. Mater. 25 (4), 1906-1915.

DOI: 10.1016/j.conbuildmat.2010.11.066

Foti D. (2013). Use of recycled waste PET bottles fibers for the reinforcement of concrete. Compos. Struct. 96, 396-404. DOI: 10.1016/j.compstruct.2012.09.019

Fraternali F., Ciancia V., Chechile R., Rizzano G., Feo L. y Incarnato L. (2011). Experimental study of the thermo-mechanical properties of recycled PET fiber-reinforced concrete. Compos. Struct. 93 (9), 2368-2374. DOI: 10.1016/j.compstruct.2011.03.025

Fraternali F., Farina I., Polzone C., Pagliuca E. y Feo L. (2013). On the use of R-PET strips for the reinforcement of cement mortars. Compos. Part B-E. 46, $207-$ 210. DOI: 10.1016/j.compositesb.2012.09.070

Fraternali F., Spadea S. y Berardi V. P. (2014). Effects of recycled PET fibres on the mechanical properties and seawater curing of Portland cement-based concretes. Constr. Build. Mater. 61, 293-302. DOI: 10.1016/j.conbuildmat.2014.03.019

Ghernouti Y., Rabehi B., Bouziani T., Ghezraoui H. y Makhloufi A. (2015). Fresh and hardened properties of self-compacting concrete containing plastic bag waste fibers (WFSCC). Constr. Build. Mater. 82, 89-100. DOI: 10.1016/j.conbuildmat.2015.02.059

Hoornweg D. y Bhada-Tata P. (2012). What a waste: a global review of solid waste management. World Bank, Washington, DC, Estados Unidos de América, 116 pp.

IRAM (2015). IRAM 1622. Cemento pórtland. Determinación de resistencias mecánicas. Instituto Argentino de Normalización y Certificación, 25 pp.

IRAM (2018). IRAM 1534. Hormigón de cemento. Preparación y curado de probetas en laboratorio para ensayos de compresión y de tracción por compresión diametral. Instituto Argentino de Normalización y Certificación, $20 \mathrm{pp}$.

INTI (2015). CIRSOC 201. Reglamento argentino de estructuras de hormigón. Instituto Nacional de Tecnología Industrial, $482 \mathrm{pp}$.

Kim J. H. J., Park C. G., Lee S. W., Lee S. J. y Won J. P. (2008). Effects of the geometry of recycled PET fiber reinforcement on shrinkage cracking of cement-based composites. Compos. Part B-E. 39 (3), 442-450. DOI: 10.1016/j.compositesb.2007.05.001

Kim S. B., Yi N. H., Kim H. Y., Kim J. H. J. y Song Y. C. (2010). Material and structural performance evaluation of recycled PET fiber reinforced concrete. Cement Concrete Comp. 32 (3), 232-240.

DOI: 10.1016/j.cemconcomp.2009.11.002

Koo B. M., Kim J. H. J., Kim S. B. y Mun S. (2014). Material and structural performance evaluations of hwangtoh admixtures and recycled PET fiber-added 
eco-friendly concrete for $\mathrm{CO}_{2}$ emission reduction. Materials 7 (8), 5959-5981. DOI: 10.3390/ma7085959

Ochi T., Okubo S. y Fukui K. (2007). Development of recycled PET fiber and its application as concretereinforcing fiber. Cement Concrete Comp. 29 (6), 448-455. DOI: 10.1016/j.cemconcomp.2007.02.002

Ojeda J., Alejandrino C. y Mercante I. (2017) Reciclaje de residuos para la elaboración de hormigones en el marco de la economía circular. Memorias. Congreso Agua, Ambiente y Energía 2017 AUGM. Mendoza, Argentina. 11, 12 y 13 de octubre, 2017, pp. 491-493.

Pereira-De-Oliveira L. A., Castro-Gomes J. P. y Nepomuceno M. C. S. (2011). Physical and mechanical behavior of recycled PET fibre reinforced mortar. Constr. Build. Mater. 25, 1712-1717.

DOI: 10.1016/j.conbuildmat.2010.11.044

Spadea S., Farina I., Carrafiello A. y Fraternali F. (2015). Recycled nylon fibers as cement mortar reinforcement. Constr. Build. Mater. 80, 200-209.

DOI: $10.1016 /$ j.conbuildmat.2015.01.075
Won J. P., Jang C. Il, Lee S. W., Lee S. J. y Kim H. Y. (2010). Long-term performance of recycled PET fibrereinforced cement composites. Constr. Build. Mater. 24 (5), 660-665. DOI: 10.1016/j.conbuildmat.2009.11.003

Yin S., Tuladhar R., Collister T., Combe M., Sivakugan N. y Deng Z. (2015a). Post-cracking performance of recycled polypropylene fibre in concrete. Constr. Build. Mater. 101, 1069-1077.

DOI: 10.1016/j.conbuildmat.2015.10.056

Yin S., Tuladhar R., Shanks R. A., Collister T., Combe M., Jacob M., Tian M. y Sivakugan N. (2015b). Fiber preparation and mechanical properties of recycled polypropylene for reinforcing concrete. J. Appl. Polym. Sci. 132 (16). DOI: 10.1002/app.41866 